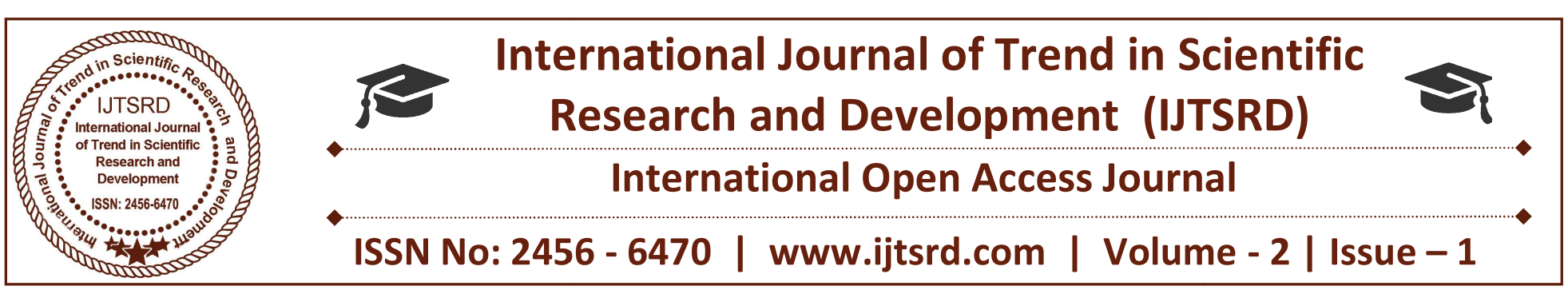

\title{
Recommendation by Service Rating Using GPS for Mobile Users
}

\author{
Prof. Brijendra Gupta, Shreyas Walujkar, Vishal Dhane, Moreshwar Tendulkar, Tushar Jadhav \\ Siddhant College of Engineering, Sudumbare, Pune
}

\begin{abstract}
Social media is for scoring system currently a day's. Users update share or tag photos throughout their visits. The geographical knowledge set by smart phone bridges the gap between physical and digital worlds. Location knowledge functions as a results of the affiliation between user's physical behaviors and virtual social net works structured by the smart phone or internet services user offers ratings thereto place and this place becomes popular the assistance of rating prediction and user is employed social media for rating. Currently a day's social media becomes fashionable. We tend to sit down with these social networks involving geographical data as location-based social networks (LBSNs). Such data brings opportunities and challenges for recommender systems to unravel the cold begin, meagerness downside of datasets and rating prediction. During this paper, we tend to alter use of the mobile users' location sensitive characteristics to hold out rating postulation. The connection between user's ratings and user-item geographical location distances, known as user-item geographical affiliation, the connection between users' rating variations and useruser geographical location distances, known as useruser geographical affiliation. Paper, we've got a bent to change use of the mobile users' location sensitive characteristics to hold out rating declaration.
\end{abstract}

Keywords: Geographical location, Rating prediction, Recommender system, Location-based social network

\section{INTRODUCTION}

With the fast development of mobile devices and omnipresent net access, social network services, like Facebook, Twitter become prevailing. in step with statistics, good phone users have created knowledge volume 10 times of a customary telephone. In 2015, there have been one.9 billion good phone users within the world, and half them had accessed to social network services. Through mobile device or on-line location primarily based social networks (LBSNs), we will share our geographical position data or check-ins. This service has attracted innumerable users. It additionally permits users to share their experiences, like reviews, ratings, photos, check-ins and moods in LBSNs with their friends. Such data brings opportunities and challenges for recommender systems. Especially, the geographical location data bridges the gap between the important world and on-line social network services. the primary generation of recommender systems with ancient cooperative filtering algorithms is facing nice challenges of cold begin for users (new users within the recommender system with very little historical records) and therefore the sparseness of datasets. If the geographical location issue is unheeded, once we search the web for a Travel, advocateer systems could recommend U.S.A. a brand new scenic spot while not considering whether or not there area unit native friends to assist U.S.A.. however if recommender systems contemplate geographical location issue, the recommendations could also be a lot of humanized and thoughtful. These area unit the motivations why we tend to utilize geographical Location data to create rating prediction.

\section{LITERATURE SURVEY}

Paper 1: Toward the next generation of recommender systems: a survey of the state-of-theart and possible extensions

Description: Author presents an overview of the world of recommender systems and describes this generation of recommendation methods that are generally classified into the following three main categories: content-based, cooperative, and hybrid 
recommendation approaches. This paper to boot describes varied limitations of current recommendation methods and discusses attainable extensions which will improve recommendation capabilities and build recommender systems applicable to a decent broader vary of applications. These extensions embrace, among others, AN improvement of understanding of users and things, incorporation of the discourse information into the recommendation method, support for multi criteria ratings, and a provision of further versatile and fewer intrusive sorts of recommendations.

Paper 2: User-Service Rating Prediction by Exploring Social Users Rating Behaviors

Description: Authors propose an inspiration of the rating schedule to represent users' daily rating behaviors. to boot, we tend to tend to propose the problem of social rating behavior diffusion to deep understand users rating behaviors. at intervals the projected user-service rating prediction approach, we tend to tend to fuse four factors, user personal interest (related to user and so the items topics), social interest similarity (related to user interest), social rating behavior similarity (related to users rating behavior habits), and social rating behavior diffusion (related to users behavior diffusions), into a unified matrixfactorized framework.

\section{Paper 3: Circle-based recommendation in online social networks}

Description: during this paper, Author presents a shot to develop circle-based RS. Author focuses on inferring category-specific social trust circles from gettable rating information combined with social network information. we tend to tend to outline several variants of weight friends within circles supported their inferred expertise levels.

\section{Paper 4: Social contextual recommendation}

Description: Author investigates social recommendation on the premise of science and science studies, that exhibit two necessary factors: individual preference and social influence. we tend to tend to initial gift the particular importance of these two factors in on-line item adoption and recommendation. Then we tend to tend to propose a singular probabilistic matrix resolving technique to fuse them in latent areas.

\section{Paper 5: Embracing Information Explosion without Choking: Clustering and Labeling in Micro blogging}

Description: during this paper, Author have an inclination to gift a text illustration framework by harnessing the power of linguistics knowledge bases, i.e., Wikipedia and WorldNet. The originally unrelated texts are connected with the linguistics illustration, thus it enhances the performance of short text cluster and labeling. The experimental results on Twitter and Facebook datasets demonstrate the superior performance of our framework in handling screaky and short little blogging messages.

\section{Paper 6: Adaptive Social Similarities for Recommender Systems}

Description: In this paper, author gift social-based technique supported matrix resolution. we tend to tend to use social knowledge to boost recommendation. The framework relies on matrix resolution with social constraint regulizer. As for social network, the similarity perform plays a significant to match the similarity between friends.

\section{Paper 7: Personalized Recommendation Combining User Interest and Social Circle}

Description: during this paper, three social factors, personal interest, social interest similarity, and social influence, fuse into a unified tailor-made recommendation model supported probabilistic matrix resolution. the problem of personal interest can produce the RS recommend things to satisfy users' individualities, significantly for delicate users. Moreover, for cold begin users, the social interest similarity and social influence can enhance the intrinsic link among choices at intervals the latent house.

\section{EXISTING SYSTEM}

The first generation of recommender systems with ancient cooperative filtering algorithms is facing nice challenges of cold begin for users (new users within the recommender system with very little historical records) and therefore the sparseness of datasets. Existing system specialise in objective analysis so as to advocate the high-quality services by exploring social user's discourse data. Except for ratings prediction, there area unit some systems that specialize in location recommendation. 
Recommender systems area unit typically classified into the subsequent classes, supported however recommendations area unit made:

Content-based recommendations: The user are going to be counseled things like those the user most popular within the past.

Collaborative recommendations:-The user are going to be counseled things that individuals with similar tastes and preferences likeable within the past.

Hybrid approaches: These strategies mix cooperative and content-based strategies.

Disadvantages of existing system

1. Ancient cooperative filtering algorithms face nice challenges of cold begin for users and therefore the sparseness of datasets.

2. Less accuracy and relevance of recommender systems.

\section{PROPOSE SYSTEM}

If recommender systems contemplate geographical location issue, the recommendations could also be a lot of humanized and thoughtful. These area unit the motivations why we tend to utilize geographical location data to create rating prediction. Recently, with the fast development of mobile devices and omnipresent net access, social network services, like
Facebook, Twitter become prevailing. In our system user visit place, if user like that place then user capture image of that place and offers rating as per their satisfaction. whereas capturing image, our system gets geographical location of that place and permit U.S.A. to share with friends / teams. In friend facet if he/she with regards to that place then he/she get notification that "One of your friend visited that specific place and counseled you to go to that place". A personalized Location based Rating Prediction (LBRP) model is projected by combining three factors: user-item geographical association, user-user geographical association, and social interest similarity. This technique user visit place, if user like that place then user capture image of that place and provides rating as per their satisfaction. Whereas capturing image, our system gets geographical location of that place and allow North yankee nation to tag express friends. In friend facet if he/she relating to that place then he/she get notification that one all told your friend visited that specific place and recommended you to travel to it place.

Advantages:

1. Our system improves the accuracy of recommender systems.

2. Our system improves applicability of recommender systems.

3. Our System more humanized and thoughtful.

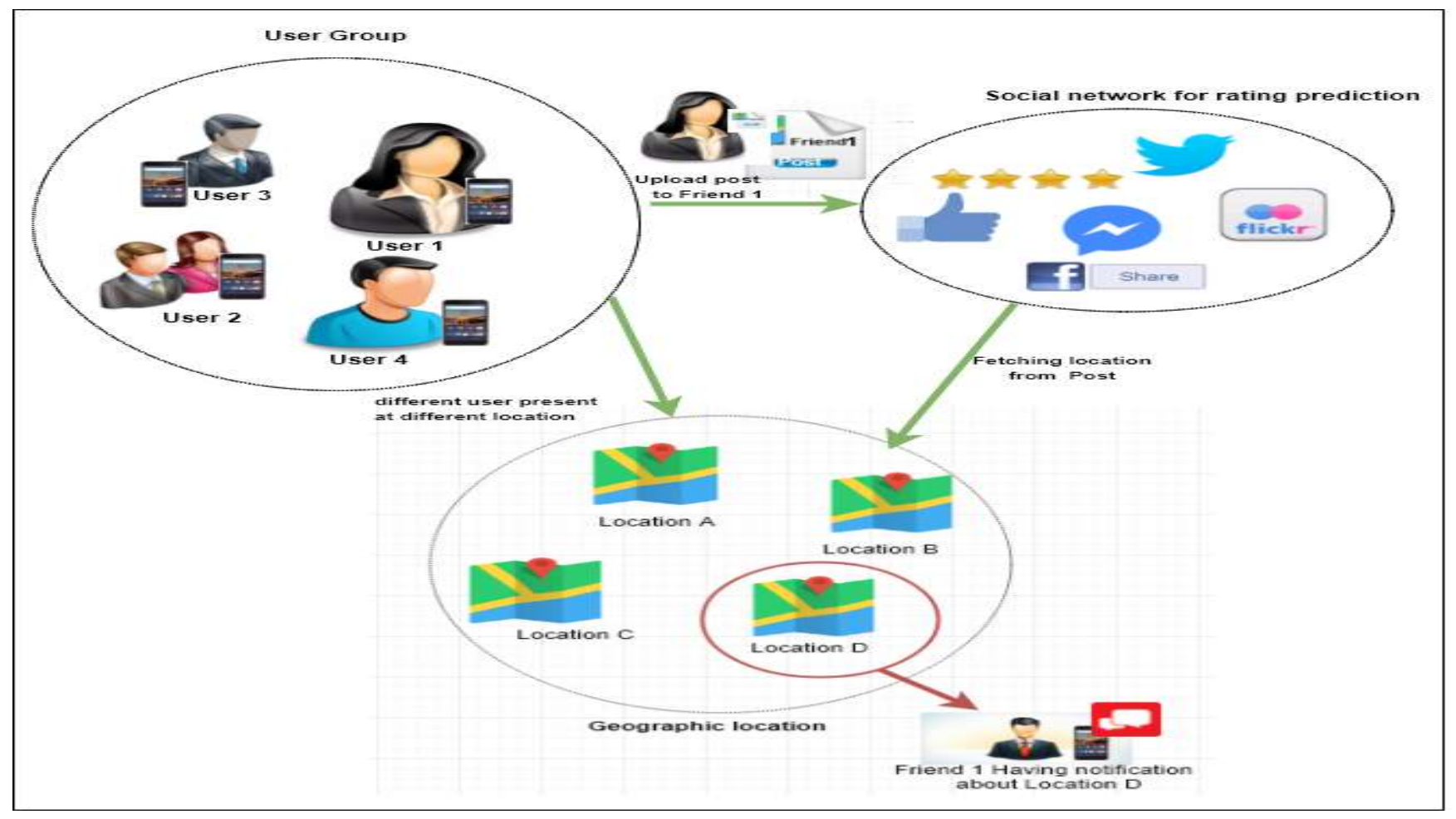




\section{ALGORITHMS}

Algorithm of location based rating prediction model LBRP

1) Initialization: $\Psi(\mathrm{t})=\Psi(\mathrm{U}(\mathrm{t}), \mathrm{P}(\mathrm{t})), \mathrm{t}=0$.

2) Set parameters: $\mathrm{k}, 1, \mathrm{n}, \lambda 1, \lambda 2, \beta, \delta, \eta$

3) Iteration:

While $(\mathrm{t}<\mathrm{n})$

Calculate $\partial \Psi / \partial \mathrm{Uu}$ And $\partial \Psi / \partial \mathrm{Pi}$

$\mathrm{U}(\mathrm{t})=\mathrm{U}(\mathrm{t})-1 \partial \Psi / \partial \mathrm{Uu} \mathrm{P}(\mathrm{t})=\mathrm{p}(\mathrm{t})-\partial \Psi / \partial \mathrm{Pi}$ $\mathrm{t}++$

4) Return: $U, P \leftarrow U(n), P(n)$

5) Prediction: $\hat{R}=\gamma+$ UT P

6) Errors: RMSE, MAE

\section{CONCLUSION}

A personalized Location based Rating Prediction (LBRP) model is projected by combining three factors: user-item geographical association, user-user geographical association, and social interest similarity. this methodology user visit place, if user like that place then user capture image of that place and provides rating as per their satisfaction. whereas capturing image, our system gets geographical location of that place and allow North yankee nation to tag specific friends. In friend side if he/she relating to that place then he/she get notification that one altogether your friend visited that specific place and recommended you to travel to it place.

\section{REFERENCES}

1) G. Adomavicius, and A. Tuzhilin, "Toward the next generation of recommender systems: a survey of the state-of-the-art and possible extensions," IEEE Transactions on Knowledge and Data Engineering, pp. 734-749, Jun. 2005.

2) X.-W. Yang, H. Steck, and Y. Liu, "Circle-based recommendation in online social networks," KDD'12, pp. 1267-1275, 2012.

3) M. Jiang, P. Cui, R. Liu, Q. Yang, F. Wang, W.-W. Zhu, and S.-Q. Yang, "Social contextual recommendation,” CIKM'12, pp. 45-54,Oct.2012.

4) $\mathrm{X} . \mathrm{Hu}, \mathrm{L}$. Tang, and H. Liu, "Embracing Information Explosion without Choking: Clustering and Labeling in Microblogging," IEEE Trans. Big Data, 2015, vol. 1, no. 1, pp. 35-46.

5) G. Zhao, X. Qian, and X. Xie, "User-Service Rating Prediction by Exploring Social Users' Rating Behaviors," IEEE Trans. Multimedia, 2016, vol.18, no.3, pp.496-506.

6) B. Sarwar, G. Karypis, J. Konstan, and J. Reidl, "Item-based collaborative filtering recommendation algorithms," World Wide Web, pp. 285-295, 2001.

7) Y. Koren, "Factorization meets the neighborhood: a multifaceted collaborative filtering model," KDD'08, 2008.

8) Y. Koren, "Collaborative filtering with temporal dynamics," KDD'09, pp. 447-456, 2009.

9) J. Wang, A. P. d. Vries, and M. J. T. Reinders, "Unifying userbased and item-based collaborative filtering approaches by similarity fusion," SIGIR'06, 2006.

10) N. N. Liu, M. Zhao, and Q. Yang, "Probabilistic latent preference analysis for collaborative filtering," CIKM'09, pp. 759-766, 2009. 